\title{
EFECTO INSECTICIDA DE SACHA YOCO (Paullinia clavigera var. bullata Simpson) (SAPINDACEAE) Y OREJA DE TIGRE (Tradescantia zebrina Hort ex Bosse) (COMMELINACEAE) EN EL CONTROL DE Anopheles benarrochi Gabaldon, Cova García y López, 1941, PRINCIPAL VECTOR DE MALARIA EN UCAYALI, PERÚ
}

\author{
INSECTICIDAL EFFECT OF Paullinia clavigera var. bullata Simpson (SAPINDACEAE) AND \\ Tradescantia zebrina Hort ex Bosse (COMMELINACEAE) IN THE CONTROL OF Anopheles benarrochi \\ Gabaldon, Cova García \& López 1941, MAIN VECTOR OF MALARIA IN UCAYALI, PERU
}

\author{
Diana Pérez ${ }^{1}$ y José Iannacone ${ }^{2}$
}

\begin{abstract}
Resumen
La resistencia de los mosquitos vectores de enfermedades metaxénicas a los insecticidas químicos, se ha incrementado en los últimos años. Frente a esta realidad, se está realizando la búsqueda de métodos alternativos, utilizando extractos de plantas con actividad larvicida. El objetivo del presente trabajo fue evaluar la mortalidad larvaria del III estadio de Anopheles benarrochi Gabaldon, Cova García \& Lopez, 1941 bajo la decocción de Paullinia clavigera var. bullata Simpson (Sapindaceae) y de la infusión de Tradescantia zebrina Hort ex Bosse (Commelinaceae). Los mayores porcentajes de mortalidad, fueron de $100 \%$ a $24 \mathrm{~h}$ de exposición a las concentraciones de 10 y $20 \%$ en $P$. clavigera y de $10 \%$ en $T$. zebrina. $P$. clavigera mostró más eficiencia insecticida sobre $A$. benarrochi en comparación con $T$. zebrina en términos de $\mathrm{CL}_{50}$ de 1 a $12 \mathrm{~h}$ de exposición; sin embargo, a las $24 \mathrm{~h}$ los valores de $\mathrm{CL}_{50}$ fueron similares $(P$. clavigera, $\mathrm{CL}_{50}=0.81 \%$ y $T$. zebrina $\mathrm{CL}_{50}=0.86 \%$ ).
\end{abstract}

Palabras clave: Anopheles benarrochi, biocida, extractos vegetales, mortalidad larvaria, Paullinia, Tradescantia.

Abstract
The resistance of mosquito vectors of metaxenic diseases to chemical pesticides has increased in the last years. Facing this reality, a search of alternative methods has begun employing plant extracts with larvicide activity. The aim of the current research was to evaluate the mortality on third stage larvae of Anopheles benarrochi Gabaldon, Cova García \& Lopez, 1941 using cooked extract of Paullinia clavigera var. bullata Simpson (Sapindaceae) and tea extract of Tradescantia zebrina Hort ex Bosse (Commelinaceae). The highest mortality percentages were $100 \%$ on $24 \mathrm{~h}$ at $10 \%$ and $20 \%$ concentrations with P. clavigera and at $10 \%$ with T. zebrina. Paullinia clavigera showed more insecticide efficient over $A$. benarrochi in comparison to $T$. zebrina in terms of $\mathrm{LC}_{50}$ at 1 to $12 \mathrm{~h}$ exposure; however, at $24 \mathrm{~h}$, the values of $\mathrm{LC}_{50}$ were similar $(P$. clavigera, $\mathrm{LC}_{50}=0.81 \%$ and $T$. zebrina $\mathrm{LC}_{50}=0.86 \%$ ).

Key words: Anopheles benarrochi, biocide, larval mortality, Paullinia, Tradescantia, vegetable extracts.

\section{Introducción}

La malaria es un problema de salud pública en numerosos países de América Latina, donde se le considera una enfermedad endémica de alta prevalencia (Iannacone \& Caballero, 1999; Blanco et al., 2000; Bobadilla et al., 2002), cuya distribución en Latinoamérica hasta el año 1999, sitúa a Brasil como el país con mayor número absoluto de casos (50.5\%), seguido por los países de la región subandina con $32.3 \%$ (OPS 2001). En la actualidad, las estrategias mundiales para prevenir y controlar la expansión de la enfermedad se basan en la utilización de insecticidas químicos en el control del vector Anopheles, pero su uso indiscriminado, ha favorecido el desarrollo de mecanismos de resistencia (Klein et al., 1991). En efecto, según la OMS (1992) en América se ha demostrado la resistencia de especies vectoras tales como Anopheles albimanus (Wiedemann, 1821),
Anopheles pseudopunctipennis Theobald, 1901, Anopheles darlingi (Root, 1926) y Anopheles vestitipennis Dyar \& Knab, 1906, a carbamatos, piretroides y organofosforados; este último grupo químico, es responsable de la resistencia en más de veinte especies de mosquitos a nivel mundial. En el Perú se emplea el temefos en los programas de control larvario, constituyendo un elemento de riesgo en salud pública debido a su toxicidad en humanos y su moderado impacto en el ambiente (Iannacone \& Alvariño, 1998).

En muchos países, el empleo de controladores biológicos ha cobrado gran relevancia y se considera con frecuencia una alternativa a los insecticidas (De Barjac, 1987). Se conoce la capacidad infectiva del hongo Beauveria bassiana (Bals-Criv) Vuill, el nemátodo Romanomermis culicivorax Ross \& Smith, 1976 (Santamarina \& Perez, 1997), la bacteria 
Bacillus thuringiensis var. israelensis H-14 (Iannacone \& Alvariño, 1997), el crustáceo Chlamydoteca sp.; así como de peces larvívoros (Iannacone \& Alvariño, 1997; Iannacone et al., 2000), entre otros, sobre larvas de anofelinos.

El empleo de productos derivados de plantas para el control de larvas de mosquitos, es una alternativa natural y es considerada segura para el medio ambiente (Iannacone et al., 2002); tal es así que se continúan investigando como repelentes en mosquitos adultos (Yang et al., 2004), y como intoxicantes e inhibidores del crecimiento frente a larvas (OPS, 1999, 2000; Iannacone et al., 2002).

La Región Ucayali cuenta con una gran diversidad de especies vegetales que, entre otros usos, pueden ser utilizadas como biocidas; sin embargo es muy limitado el conocimiento y uso de estas especies. Mediante estudios etnobotánicos en Ucayali, Paullinia clavigera Simpson y Tradescantia zebrina Hort ex Bosse han sido seleccionadas con potencial biocida para ser investigadas en el control de los vectores de la malaria, especialmente en $A$. benarrochi considerado en Ucayali el vector primario (Pérez, 2002; Schoeler et al., 2003).

Las poblaciones naturales de $P$. clavigera se ubican en ecosistemas de altura Amazónica, formando parte del bosque primario y en planicies anegadizas, requieren de poca luminosidad, de moderada a alta humedad relativa y de altitudes de 150 a $2000 \mathrm{msnm}$. Prefieren los suelos arcillosos. Su raíz se utiliza como ictiotóxico para la pesca, y presenta actividad antifúngica y molusquicida. El contenido de taninos (principalmente ácido catecutánico y catecol) es muy alto, por lo que provoca efectos en el sistema nervioso central; asimismo presenta una gran cantidad de cafeína que varía de 3 a $5 \%$ del peso seco, y una saponina llamada timbonina con propiedades ictiotóxicas, se encuentra en cantidades pequeñas. En la especie congenérica a $P$. clavigera, Paullinia pinnata se han registrado actividades molusquicidas contra Biomphalaria glabrata, hospedero intermediario de Schistosoma mansoni (Melendez \& Carriles, 2002), posiblemente debido a flaconas glicosiladas (Abourashed et al., 1999). En Paullinia cupana "Guarana" se ha evaluado su importancia como remedio herbal natural y etnobotánico (Myerscough, 1998) y se ha evaluado la toxicidad del extracto acuoso sobre células de ovario de hamster y sobre la bacteria Vibrio fisheri, encontrándose que dosis altas pudieran ser dañinas para la salud humana (Santa María et al., 1998). Extractos acuosos de $P$. cupana han mostrado actividad genotóxica y mutagénica en células bacterias de Escherichia coli (da Fonseca et al., 1994). Altas concentraciones de cafeína se han encontrado en Paullina yoco y $P$. cupana (Schutter, 1994; Cipollini, 2000). Plotkin (1988) señala a $P$. cupana como una fuente potencial de plaguicida biodegradable.
En el caso de $T$. zebrina se le encuentra en ecosistemas de Bosque húmedo tropical, en suelos franco limosos con abundante materia orgánica, no tolera inundaciones prolongadas, ni exposición directa al sol. Se reporta que la hoja por contacto puede desencadenar picazón tóxica, con propiedades cáusticas e inflamatorias a la piel. La planta posee principalmente antocianinas. Se le considera una planta de importancia ornamental; se le ha usado para la detección de radiaciones y para el biomonitoreo de la calidad del aire para detectar metales trazas (Isidoro et al., 2003; Kim et al., 2003; Sumita et al., 2003).

Bajo estas consideraciones, el objetivo del presente trabajo fue evaluar in vitro la mortalidad de larvas de $A$. benarrochi empleando el extracto por decocción de $P$. clavigera var. bullata y por infusión de T. zebrina.

\section{Materiales y métodos \\ Mosquitos adultos}

Se colectaron los mosquitos en estado adulto según el protocolo propuesto por Macedo et al. (1997) intradomiciliariamente, con cebo animal, en el caserío San José del distrito de Campo Verde, provincia de Coronel Portillo, departamento de Ucayali, Perú. Zona de alto riesgo endémico para malaria. Luego se trasladaron al Laboratorio de Entomología del Instituto de Investigaciones de la Amazonia Peruana (IIAP), filial Ucayali, ubicado en la carretera Federico Basadre (CFB) km 12.400. La especie A. benarrochi se identificó a nivel del estadio adulto usando las claves de determinación taxonómica (Lounibos et al., 1997; Sallum et al., 1997; Calle et al., 2002).

\section{Crianza de larvas}

Se siguió las recomendaciones de Macedo et al. (1997), usando fuentes de porcelana de $40 \times 28$ × $5 \mathrm{~cm}$ con agua de criadero artificial, la cual se adicionó interdiariamente. La alimentación a base de materia orgánica fue provista ad libitum mediante la planta acuática Pistia stratiotes L., las cuales fueron introducidas en el medio de crianza, así como por microorganismos en suspensión provenientes del agua de criadero.

\section{Extractos botánicos}

Las lianas de $P$. clavigera (PC) fueron colectadas en el km 83 de la CFB, Caserío "Señor de los Milagros", jurisdicción del distrito de Irazola, Provincia de Padre Abad y T. zebrina (TZ) fue obtenida del Banco de Genes de Plantas Biocidas y Medicinales del Instituto de Investigaciones de la Amazonia Peruana (IIAP), Ucayali, ubicada en la CFB $\mathrm{km}$ 12.400. Las lianas de PC fueron secadas directamente al sol por espacio de dos semanas aproximadamente y se trituraron en un molino de martillo. Se pesaron $250 \mathrm{~g}$ de PC y se realizó la decocción con $2.5 \mathrm{~L}$ de agua destilada, la cual fue estandarizada a pH 7 con $\mathrm{NaON} 1 \mathrm{~N}$, la decocción se realizó por espacio de $2 \mathrm{~h}$ hasta obtener un $\mathrm{L}$ de solución madre de color rojo tinto. Para el caso de TZ 
se secó toda la planta directamente al sol hasta perder el $90 \%$ de humedad aproximadamente, se trituró en un molino de martillo; se realizó una infusión con 100 g de TZ triturada en $1 \mathrm{~L}$ de agua destilada a $80{ }^{\circ} \mathrm{C}$, del cual se obtuvo $800 \mathrm{ml}$ de solución madre.

Bioensayos

Se utilizaron larvas del III estadio de $A$. benarrochi, los cuales se expusieron al extracto de PC por decocción y a TZ por infusión. Se prepararon concentraciones al $5 \%, 10 \%, 15 \%$ y $20 \%$ para PC y concentraciones de $2.5 \%, 5 \%, 7.5 \%$ y $10 \%$ para TZ ([peso seco en $\mathrm{g}$ de la planta/ volumen de agua destilada] x 100), los cuales correspondieron a agregar $20 ; 40 ; 60 ;$ y $80 \mathrm{ml}$ del extracto patrón (Solución Madre) de ambas especies de plantas, y enrasados a $100 \mathrm{ml}$ con agua destilada. Se emplearon vasos de plástico circulares descartables de $250 \mathrm{ml}$ de capacidad. Todos los bioensayos se realizaron a una temperatura no controlada de $30{ }^{\circ} \mathrm{C} \pm 5{ }^{\circ} \mathrm{C}$ y humedad relativa entre 60 y $85 \%$.

\section{Evaluación de la mortalidad}

Las lecturas de mortalidad de $A$. benarrochi se realizaron a $1,4,8,12$ y $24 \mathrm{~h}$ de exposición. Las larvas se consideraron muertas cuando no reaccionaron al momento de ser tocadas con un puntero romo en la región cervical durante $10 \mathrm{seg}$. de observación (Macedo et al., 1997).

\section{Análisis estadístico}

Las pruebas de toxicidad aguda de los extractos acuosos sobre $A$. benarrochi se evaluaron en cuatro concentraciones más el control, con cuatro
Tabla 1. Condiciones y criterios de aceptabilidad de la prueba de toxicidad aguda con Anopheles benarrochi

\begin{tabular}{|c|c|}
\hline Tipo de bioensayo & estático \\
\hline Tiempo de exposición & 1. $4,8,12$ y $24 \mathrm{~h}$ \\
\hline Temperatura & $29 \pm 6^{\circ} \mathrm{C}$ \\
\hline Humedad relativa & $60-85 \%$ \\
\hline pH de la solución & 7 \\
\hline Fotoperiodo & $12: 12$ \\
\hline Tamaño de envase & capacidad de $250 \mathrm{ml}$ \\
\hline Volumen de la solución / envase & $100 \mathrm{ml}$ \\
\hline Edad de organismos & larvas del III estadio \\
\hline $\mathrm{N}^{\mathrm{o}}$ de réplicas / concentración & 4 \\
\hline $\mathrm{N}^{\mathrm{o}}$ de concentraciones más & 40 \\
\hline control & 5 \\
\hline $\mathrm{N}^{\mathrm{o}}$ de larvas / concentración & 10 \\
\hline $\mathrm{N}^{\mathrm{o}}$ de larvas por envase & 200 \\
\hline $\mathrm{N}^{o}$ total de larvas por ensayo & no requiere \\
\hline Régimen de alimentación & destilada \\
\hline Agua control y de dilución & Directa por $10 \mathrm{seg}$. \\
\hline Tiempo de observación & mortalidad (cuando \\
\hline Respuesta letal & $\begin{array}{l}\text { reaccionan al con algún } \\
\text { movimiento al ser tocadas en } \\
\text { la región cervical). }\end{array}$ \\
\hline & $\begin{array}{l}\text { Sobre } 80 \% \text { de sobrevivencia } \\
\text { en los controles. }\end{array}$ \\
\hline
\end{tabular}

Tabla 2. Efecto de extractos acuosos de Paullinia clavigera en la mortalidad de larvas de Anopheles benarrochi en bioensayos de laboratorio a diferentes horas de evaluación.

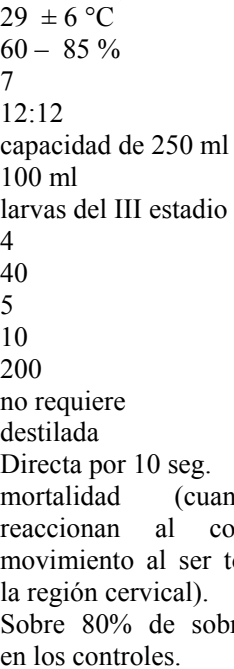
repeticiones, en un diseño de Bloque Completo al Azar (DBCA): $5 \mathrm{x}$ 4. La eficacia de los tratamientos y las repeticiones se evaluó a través de un análisis de varianza (ANDEVA) de dos vías, previa transformación de los datos a raíz cuadrada del arcoseno. En el

\begin{tabular}{cc}
\hline $\begin{array}{c}\text { Conœentración } \\
(\%)\end{array}$ & $\begin{array}{c}1 \mathrm{~h} \\
\% \\
\text { Mbrtalic }\end{array}$ \\
\hline Agua destilada & 0 \\
\hline 5 & 0 \\
10 & 15 \\
15 & 22,5 \\
20 & 42,5 \\
\hline
\end{tabular}
caso de existir diferencias significativas entre los tratamientos y entre las repeticiones se realizó la prueba de Tukey. Los cálculos de la mortalidad corregida se realizaron mediante la fórmula de Abbott en caso de muerte natural en el grupo testigo cuando éste era menor al $20 \%$ (Macedo et al., 1997). Los datos se analizaron mediante el paquete SAS Institute Inc, 1989. Las concentraciones letales medias $\left(\mathrm{CL}_{50}\right)$, así como sus límites de confianza se determinaron utilizando el programa de la EPA Probit.

\section{Resultados y discusión}

Las principales condiciones y criterios de aceptabilidad para la prueba de toxicidad aguda con las formas larvarias del mosquito $A$. benarrochi, empleando extractos de $\mathrm{PC}$ por decocción y de $\mathrm{TZ}$ por infusión, mostraron un $80 \%$ de sobrevivencia en los controles (Tabla 1). 
Tabla 3. Efecto de extractos acuosos de Tradescantia zebrina en la mortalidad de larvas de Anopheles benarrochi en bioensayos de laboratorio a diferentes horas de evaluación.

\begin{tabular}{|c|c|c|c|c|c|c|}
\hline \multirow[b]{2}{*}{$\begin{array}{c}\text { Concentración } \\
(\%)\end{array}$} & \multicolumn{2}{|l|}{1 hora } & \multicolumn{2}{|c|}{4 horas } & \multicolumn{2}{|l|}{8 horas } \\
\hline & $\begin{array}{c}\% \\
\text { Mortalidad }\end{array}$ & Sign. & $\begin{array}{c}\% \\
\text { Mortalidad }\end{array}$ & Sign & $\begin{array}{c}\% \\
\text { Mortalidad }\end{array}$ & Sign \\
\hline Agua destilada & 0 & $a$ & 0 & $a$ & 0 & $\mathrm{~b}$ \\
\hline 2,5 & 0 & $a$ & 0 & $a$ & 0 & $\mathrm{~b}$ \\
\hline 5 & 0 & a & 0 & a & 0 & $b$ \\
\hline 7,5 & 0 & a & 0 & a & 10 & $b$ \\
\hline 10 & 0 & a & 0 & a & 42,5 & a \\
\hline \multicolumn{7}{|c|}{$\begin{array}{l}\text { Promedio en una misma línea vertical seguidos por la misma letra } \\
\text { minúscula no difieren significativamente a } \mathrm{P}=0.05 \text {. Prueba de } \\
\text { Tukey (SAS, 1989). } \\
\text { Sign.= Significancia. } \\
\text { Valores entre paréntesis están corregidos por la fórmula de Abbott. } \\
\text { Los valores de las concentraciones en ml fueron transformados a \% } \\
\text { ([peso seco de la planta/volumen de agua destilada] x 100). }\end{array}$} \\
\hline
\end{tabular}

A diferencia de PC, Tradescantia inicia recién su efecto biocida a $8 \mathrm{~h}$ de exposición, en forma significativa con respecto al testigo (agua destilada), en concentración del $10 \%$; observándose a las $12 \mathrm{~h}$ y a las $24 \mathrm{~h}$ mortalidades significativas a las diferentes concentraciones (Tabla 3).

El PC presenta mayor efecto insecticida que $\mathrm{TZ}$ de 1 a $12 \mathrm{~h}$ de exposición. Sin embargo, a $24 \mathrm{~h}$ de exposición los valores de $\mathrm{CL}_{50}$ son numéricamente semejantes (Tabla 4).

Tabla 4. Valores de la concentración letal media $\left(\mathrm{CL}_{50}\right)$ en los extractos acuosos de Paullinia clavigera y Tradescantia zebrina sobre larvas de Anopheles benarrochi.

\begin{tabular}{ccc} 
benarrochi. & \multicolumn{2}{c}{ CL $_{50}(\%)$} \\
\hline $\begin{array}{c}\text { Periodo de } \\
\text { exposición }(\mathrm{h})\end{array}$ & Paullinia clavigera & Tradescantia zebrina \\
\hline 1 & $25.46(21.54-30.09)$ & $>17.62$ \\
4 & $11.21(9.55-13.16)$ & $>17.62$ \\
8 & $6.17(5.43-7.02)$ & $17.62(14.35-21.63)$ \\
12 & $1.99(1.36-2.91)$ & $7.23(6 . .53-8.02)$ \\
24 & $0.81(0 . .55-1.18)$ & $0.86(0.52-1.40)$ \\
\hline
\end{tabular}

Más de 2000 especies de plantas poseen químicos con propiedades biocidas en el control de plagas, y entre éstas, 344 especies han demostrado que tienen algún grado de actividad contra las larvas de mosquitos (Sukumar et al., 1991). La Tabla 5 muestra 54 plantas pertenecientes a 30 familias con propiedades larvicidas contra culícidos reportadas en la literatura mundial en los últimos seis años (entre 1998 al 2003).

La efectividad de los insecticidas vegetales es dependiente de algunos factores extrínsecos, tales como la especie y variedad de la planta, época de recolección, parte cosechada y forma de preparación, extracción y aplicación (Iannacone et al., 2002). Además Amadiola (2000), señala que las diferencias en la toxicidad de diferentes extractos pudieran deberse a la solubilidad de sus compuestos activos en los solventes o a la presencia de inhibidores activos en los solventes o a la presencia de inhibidores de principios insecticidas. En el presente estudio, en ambas especies de plantas se empleó agua destilada como solvente.

\begin{tabular}{|c|c|c|c|}
\hline \multicolumn{2}{|c|}{12 horas } & \multicolumn{2}{|c|}{24 horas } \\
\hline $\begin{array}{c}\% \\
\text { Mortalidad }\end{array}$ & Sign. & $\begin{array}{c}\% \\
\text { Mortalidad }\end{array}$ & Sig. \\
\hline 0 & $\mathrm{~b}$ & $15(0)$ & $\mathrm{C}$ \\
\hline 0 & $\bar{b}$ & $65(58,82)$ & $\mathrm{b}$ \\
\hline 17,5 & b & $90(88,24)$ & $a b$ \\
\hline 52,5 & a & $95(94,12)$ & $a b$ \\
\hline 80 & a & $100(100)$ & a \\
\hline
\end{tabular}

Entre los factores inherentes al organismo de prueba, es de destacar la variación de la susceptibilidad de acuerdo a la edad, estado de desarrollo, reorganización anatómica y a las variaciones propias de la muda; existe además, una tasa metabólica muy baja en individuos cercanos a la pupación, como las larvas; tal es así que se prefirió trabajar con el III estadio, debido a que Mulla \& Su (1999) muestran que este estadio fue el más susceptible en comparación con el IV (Xue et al., 2000).

De otro lado, la actividad tóxica de los principios activos de las plantas biocidas empleadas sería de ingesta y de contacto (Stoll, 1989; Rodríguez, 2000). De ingesta, porque al alimentarse las larvas mediante filtración y al no poseer una ingestión selectiva de partículas, los larvicidas pueden ingresar libremente produciendo toxicidad digestiva (Macedo et al., 1997); y de contacto, mediante tres mecanismos interdependientes: transporte desde la cutícula al sitio de acción, inhibición enzimática y efecto sobre el sistema nervioso central, respiratorio u otro sistema involucrado como una consecuencia bioquímica del primer mecanismo (Gunther \& Jeppson, 1962).

Se concluye que ambas especies de plantas $P$. clavigera y $T$. zebrina son candidatas ideales y promisorias como agentes biocidas naturales para el control larvario de $A$. benarrochi, debido a que producen $\mathrm{CL}_{50} \mathrm{~s}$ de $0.81 \%$ a $0.86 \%$ a las $24 \mathrm{~h}$ de exposición (Tablas 2 y 3 ).

\section{Literatura citada}

Abourashed E.A., Toyang N.J., Choinski J.Jr. \& Khan I.A. 1999. Two new flavone glycosides from Paullinia pinnata. J. Nat. Prod. 62: 1179-1181.

Amadiola A.C. 2000. Controlling rice blast in vitro and in vivo with extracts of Azadiracta indica. Crop Protection. 19: 452-460.

Araujo E.C., Silveira E.R., Lima M.A., Neto M.A., de Andrade I.L., Santiago G.M. \& Mesquita A.L. 2003. Insecticidal activity and chemical composition of volatile oils from Hyptis martiusii Benth. J. Agric. Food Chem. 51: 3760-3762.

Bandara K.A., Kumar V., Jacobsson U. \& Molleyres L.P. 2000. Insecticidal piperidine alkaloid from Microcos paniculata stem bark. Phytochemistry. 54: 29-32.

Blanco S., Martínez A., Cano O., Tello R. \& Mendoza E.I. 2000. Introducción al Bacillus sphaericus cepa-2362 (GRISELEF) para el control biológico 
de vectores maláricos en Guatemala. Rev. Cub. Med. Trop. 52: 37-43.

Bobadilla A.M., Zavaleta G., Franco F.G. \& Pollack, L. 2002. Efecto bioinsecticida del extracto etanólico de las semillas de Annona cherimolia Miller "chirimoya" y $A$. muricata Linneaus "guanabana" sobre larvas del IV estadio de Anopheles sp. Rev. peru. biol. 9: 64 -73.

Calderón G., Fernández R. \& Valle J. 1995. Especies de la fauna anofelina, su distribución y algunas consideraciones sobre su abundancia e infectividad en el Perú. Rev. peru. Epidemiol. 8: 5-23.

Calle L.D.A., Quinones M.L., Erazo H.F. \& Jaramillo O.N. 2002. Morphometric discrimination of females of five species of Anopheles of the subgenus Nyssorhynchus from Southern and Northwest Colombia. Mem. Inst. Oswaldo Cruz. 97: 1191-1195.

Chariandy C.M., Seaforth C.E., Phelps R.H., Pollard G.V. \& Khambay B.P. 1999. Screening of medicinal plants from Trinidad and Tobago for antimicrobial and insecticidal properties. $\mathrm{J}$. Ethnopharmacol. 64: 265-270.

Ciccia G., Coussio J. \& Mongelli E. 2000. Insecticidal activity against Aedes aegypti larvae of some medicinal South American plants. J. Ethnopharmacol. 72: 185-189.

Cipollini M.L. 2000. Secondary metabolitos of vertebrate-dispersed fruits: evidence for adaptative functions. Rev. Chil. hist. Nat. 73: 421-440.

da Fonseca C.A., Leal J., Costa S.S. \& Leitao A.C. 1994. Genotoxic and mutagenic effects of guarana (Paullinia cupana) in prokaryotic organisms. Mutat. Res. 321: 165-73.

David J.P., Rye D., Pautou M.P. \& Meyran J.C. 2000. Differential toxicity of leaf litter to dipteran larvae of mosquito developmental sites. J. Invertebr. Pathol. 75: 9-18.

De Barjac H. 1987. Operational bacterial insecticides and their potential for future improvement. Appl. Microbiol. Biotechnol. 21: 85-90.

Diallo D., Marston A., Terreaux C., Toure Y., Paulsen B.S. \& Hostettmann K. 2001. Screening of Malian medicinal plants for antifungal, larvicidal, molluscicidal, antioxidant and radical scavenging activities. Phytother. Res. 15: 401-406.

El Hag E.A., El Nadi A.H. \& Zaitoon A.A. 1999. Toxic and growth retarding effects of three plant extracts on Culex pipiens larvae (Diptera: Culicidae). Phytother. Res. 13: 388-392.

Gunther A. \& Jeppson L. 1962. Insecticidas modernos y la producción mundial de alimentos. Compañía Editorial Continental S. A. México D. F.

Hung H. S., Wang J., Sim K.Y., Ee G.C., Imiyabir Z., Yap K.F., Shaari K., Hock K. \& Goh S. 2003. Meliternatin: a feeding deterrent and larvicidal polyoxygenated flavone from Melicope subunifoliolata. Phytochemistry. 62: 1121-1124.
Iannacone J. \& Alvariño L. 1997. Peces larvívoros con potencial para el control biológico de estados inmaduros de zancudos en el Perú. Rev. per. Ent. 40: 9-19.

Iannacone J. \& Alvariño L. 1998. Ecotoxicidad aguda del insecticida organofosforado temephos sobre Chironomus calligraphus Goeldi (Diptera: Chironomidae). Acta Entomol. Chilena. 22: 53-55.

Iannacone J. \& Caballero C. 1999. La técnica de precoloración de Walker para evaluar Plasmodium vivax Grassi y Plasmodium malariae Leveran en comunidades Asháninkas en Satipo (Junín, Perú). Rev. per. Biol. 6: 171-180.

Iannacone J., Alvariño L., Moreno R., Reyes M. \& Chauca J. 2000. Culícidos (Diptera) en la provincia Constitucional del Callao, Perú, durante el Niño 1997-98. Acta Entomol. Chilena. 24: 5160 .

Iannacone J., Alvariño L. \& Mansilla J. 2002. Actividad insecticida de cuatro extractos botánicos sobre larvas de los mosquitos Culex quinquefasciatus (Díptera: Culicidae) y Chironomus calligraphus (Díptera:Chironomidae). Wiñay Yachay (Perú). 6: 56 - 71.

Ioset J.R., Marston A., Gupta M.P. \& Hostettmann K. 2000. Antifungal and larvicidal cordiaquinones from the roots of Cordia curassavica. Phytochemistry. 53: 613-617.

Ioset J.R., Marston A., Gupta M.P. \& Hostettmann K. 2001. Five new prenylated stilbenes from the root bark of Lonchocarpus chiricanus. J. Nat. Prod. 64: 710-715.

Isidoro M., Ferrara M., Lavorgna M., Nardelli A. \& Parrilla A. 2003. In situ monitoring of urban air in Southern Italy with the Tradescantia micronucleus bioassay and semipermeable membrane devices (SPMDs). Chemosphere. 52:121-126.

Jang Y.S., Baek B.R., Yang Y.C., Kim M.K. \& Lee H.S. 2002. Larvicidal activity of leguminous seeds and grains against Aedes aegypti and Culex pipiens pallens. J. Am. Mosq. Control Assoc. 18: 210-213.

Jaswanth A., Ramanathan P. \& Ruckmani K. 2002. Evaluation of mosquitocidal activity of Annona squamosa leaves against filarial vector mosquito, Culex quinquefasciatus Say. Indian J. Exp. Biol. 40: 363-365.

Jeyabalan D., Arul N. \& Thangamathi P. 2003. Studies on effects of Pelargonium citrosa leaf extracts on malarial vector, Anopheles stephensi Liston. Bioresour. Technol. 89: 185-189.

Kim J.K., Shin H.S., Lee J.H., Lee J.J. \& Lee J.H. 2003. Genotoxic effects of volatile organic compounds in a chemical factory as evaluated by the Tradescantia micronucleus assay and by chemical analysis. Mutat. Res. 541: 55-61.

Klein T.A., Lima J.B., Tada M.S. \& Miller R. 1991. Comparative susceptibility of anopheline mosquitoes in Rondonia, Brazil to infection by 
Plasmodium vivax. Am. J. Trop. Med. Hyg. 45: 463-470.

Lee S.E. 2000. Mosquito larvicidal activity of pipernonaline, a piperidine alkaloid derived from long pepper, Piper longum. J. Am. Mosq. Control Assoc. 16: 245-247.

Lounibos L.P., Duzak D. \& Linley J.R. 1997. Comparative egg morphology of six species of the albimanus section of Anopheles (Nyssorhynchus) (Diptera: Culicidae). J. Med. Entomol. 34: 136155.

Macedo M.E., Consoli R.A., Grande T.S., dos Anjos A.M., de Oliveira A.B., Mendes N.M., Queiroz R.O. \& Zani C.L. 1997. Screening of Asteraceae (Compositae) plant extracts for larvicidal activitry against Aedes fluviatilis (Diptera: Culicidae). Mem. Inst. Oswaldo Cruz. 92: 565-570.

Mansour S.A., Messeha S.S. \& el-Gengaihi S.E. 2000. Botanical biocides. 4. Mosquitocidal activity of certain Thymus capitatus constituents. J. Nat. Toxins. 9: 49-62.

Melendez P.A. \& Carriles V.A. 2002. Molluscicidal activity of plants from Puerto Rico. Ann. Trop. Med. Parasitol. 96: 209-218.

Miles J.E., Ramsewak R.S. \& Nair M.G. 2000. Antifeedant and mosquitocidal compounds from Delphinium $x$ cultorum cv. Magic fountains flowers. J. Agric. Food Chem. 48: 503-506.

Momin R.A. \& Nair M.G. 2001. Mosquitocidal, nematicidal, and antifungal compounds from Apium graveolens L. seeds. J. Agric. Food Chem. 49: 142-145.

Momin R.A. \& Nair M.G. 2002. Pest-managing efficacy of trans-asarone isolated from Daucus carota L. seeds. J.Agric.Food Chem. 50: 44754478.

Mongelli E., Coussio J. \& Ciccia G. 2002. Investigation of the larvicidal activity of Pothomorphe peltata and isolation of the active constituent. Phytother. Res. 16 Suppl 1: S71-S72.

Mulla M.S. \& Su T. 1999. Activity and Biological effects of neem products against arthropods of medical veterinary importance. J. Am. Mosq. Control Assoc. 16: 234-240

Myerscough M. 1998. Herbal remedies. How much do you know? Aust. Fam. Physician. 27: 1037-1040.

Ndung'u M., Hassanali A., Hooper A.M., Chhabra S., Miller T.A., Paul R.L. \& Torto B. 2003. Ring Aseco mosquito larvicidal limonoids from Turraea wakefieldii. Phytochemistry. 64: 817-823.

Oberlies N.H., Rogers L.L., Martin J.M. \& McLaughlin J.L. 1998. Cytotoxic and insecticidal constituents of the unripe fruit of Persea americana. J. Nat. Prod. 61: 781-785.

ORGANIZACIÓN MUNDIAL DE LA SALUD (OMS). 1992. Resistencia de los Vectores de Enfermedades a los Plaguicidas. 15. ${ }^{\circ}$ Informe del Comité de Expertos de la OMS en Biología de
Vectores y Lucha Antivectorial (Serie de Informes Técnicos N. ${ }^{\circ}$ 818). Ginebra.

ORGANIZACIÓN PANAMERICANA DE LA SALUD (OPS). 1999. Control Selectivo de Vectores de Malaria. Guía para el nivel local de los sistemas de salud. Washington, DC. IV.

ORGANIZACIÓN PANAMERICANA DE LA SALUD (OPS). 2001. Situación de los programas de malaria en las Américas. Bol. Epid. Org. Panam. Salud. 22:10-14.

Park I.K., Lee S.G., Shin S.C., Park J.D. \& Ahn Y.J. 2002. Larvicidal activity of isobutylamides identified in Piper nigrum fruits against three mosquito species. J. Agric. Food Chem. 50: 18661870.

Pelah D., Abramovich Z., Markus A. \& Wiesman Z. 2002. The use of commercial saponin from Quillaja saponaria bark as a natural larvicidal agent against Aedes aegypti and Culex pipiens. J. Ethnopharmacol. 81: 407-409.

Pérez D. 2002. Etnobotánica medicinal y biocidas para malaria en la región Ucayali. Folia Amazoniana. 13: 85-106.

Pitasawat B., Choochote W., Kanjanapothi D., Panthong A., Jitpakdi A. \& Chaithong U. 1998. Screening for larvicidal activity of ten carminative plants. Southeast Asian J. Trop. Med. Public Health. 29: 660-662.

Plotkin M. 1988. The outlook for new agricultural and industrial products from the tropics: Natural pesticides. In Biodiversity, ed. E.O. Wilson, 11112. Washington, D.C.: National Academy Press.

Rahuman A.A., Gopalakrishnan G., Ghouse B.S., Arumugam S. \& Himalayan B. 2000. Effect of Feronia limonia on mosquito larvae. Fitoterapia. 71: 553-555.

Ramsewak R.S., Nair M.G., Murugesan S., Mattson W.J. \& Zasada J. 2001. Insecticidal fatty acids and triglycerides from Dirca palustris. J Agric Food Chem. 49: 5852-5856.

Redwane A., Lazrek H.B., Bouallam S., Markouk M., Amarouch H. \& Jana M. 2002. Larvicidal activity of extracts from Quercus lusitania var. infectoria galls (Oliv.). J. Ethnopharmacol. 79: 261-263.

Rodríguez C. 2000. Plantas Contra Plagas: Potencial práctico de ajo, anona, nim, chile y tabaco. Ed. RAPAM. México. D. F.

Sallum M., Bergo E.S. \& Forattini O.P. 1997. First record of Anopheles benarrochi Gabaldon, Cova García \& Lopez from the State of Sao Paulo, Southern Brazil. Mem. Inst. Oswaldo Cruz. 92: 233-234.

Santa Maria A., Lopez A., Diaz M.M., MunozMingarro D. \& Pozuelo J.M. 1998. Evaluation of the toxicity of guarana with in vitro bioassays. Ecotoxicol. Environ. Saf. 39: 164-167.

Santamarina M.A. \& Perez P.R. 1997. Reduction of mosquito larval densities in natural sites after 
introduction of Romanomermis culicivorax (Nematoda: Mermithidae) in Cuba. J. Med. Entomol. 34: 1-4.

Schoeler G.B., Flores-Mendoza C., Fernandez R., Davila J.R. \& Zyzak M. 2003. Geographical distribution of Anopheles darlingi in the Amazon Basin region of Peru. J. Am. Mosq. Control Assoc. 19: 286-296.

Schultes R.E. 1994. Amazonian ethnobotany and the search for new drugs. Ciba Found Symp. 185: 106112; discussion: 112-115.

Siddiqui B.S., Afshan F., Gulzar T., Sultana R., Naqvi S.N. \& Tariq R.M. 2003. Tetracyclic triterpenoids from the leaves of Azadirachta indica and their insecticidal activities. Chem. Pharm. Bull. (Tokyo). 51: 415-417.

Stoll G. 1989. Protección natural de cultivos con recursos provenientes de las granjas de las zonas tropicales y sub tropicales. Ed. Científica José Margraf. Alemania.

Sukumar K., Perich J. \& Boombar L.R. 1991. Botanical derivatives in mosquito control; A review. J. Am. Mosq. Control. Assoc. 7: 210-237.

Sumita N.M., Mendes M.E., Macchione M., Guimaraes E.T., de Lichtenfels A.J., de Lobo D.J., Saldiva P.H. \& Saiki M. 2003. Tradescantia pallida $\mathrm{cv}$. purpurea boom in the characterization of air pollution by accumulation of trace elements. J. Air Waste Manag. Assoc. 53:574-579.

Sun R., Sacalis J.N., Chin C.K. \& Still C.C. 2001. Bioactive aromatic compounds from leaves and stems of Vanilla fragrans. J. Agric. Food Chem. 49: 5161-5164.

Tiew P., Ioset J.R., Kokpol U., Chavasiri W. \& Hostettmann K. 2003. Antifungal, antioxidant and larvicidal activities of compounds isolated from the heartwood of Mansonia gagei. Phytother. Res. 17: 190-193.

Traboulsi A.F., Taoubi K., el-Haj S., Bessiere J.M. \& Ramal S. 2002. Insecticidal properties of essential plant oils against the mosquito Culex pipiens molestus (Diptera: Culicidae). Pest. Manag. Sci. 58: 491-495.

Vahitha R., Venkatachalam M.R., Murugan K. \& Jebanesan A. 2002. Larvicidal efficacy of Pavonia zeylanica L. and Acacia ferruginea D.C. against Culex quinquefasciatus Say. Bioresour. Technol. 82: 203-204.

Xue R.D., Barnard D.R. \& Ali A. 2000. Laboratory and field comparison of pyriproxyfen, polystyrene beads and other larvicidal methods against malaria vectors in Sri Lanka. Acta Trop. 81: 211-233.

Yang Y.C., Lee S.G., Lee H.K., Kim M.K., Lee S.H. \& Lee H.S. 2002. A piperidine amide extracted from Piper longum L. fruit shows activity against Aedes aegypti mosquito larvae. J. Agric. Food Chem. 50: 3765-3767.

Yang Y.C., Lee E.H., Lee H.S., Lee D.K. \& Ahn Y.J. 2004. Repellency of aromatic medicinal plant extracts and a steam distillate to Aedes aegypti. J. Am. Mosq. Control Assoc. 20: 146-149.

\section{Tabla citada en el texto}

Tabla 5. Plantas con propiedades biocidas empleadas para el control de mosquitos culícidos entre 1998 al 2003.

\begin{tabular}{|c|c|c|c|c|}
\hline Familia & Nombre científico & $\begin{array}{l}\text { Parte de } \\
\text { la planta } \\
\end{array}$ & Especie de mosquito & Referencia \\
\hline Acanthaceae & $\begin{array}{l}\text { Justicia pectoralis Vault } \\
\text { Glinus oppositifolius (L.) }\end{array}$ & hojas & Aedes aegypti (L.) & Chariandy et al. (1999) \\
\hline Aizoaceae & $\begin{array}{l}\text { D.C. } \\
\text { Glinus oppositifolius (L.) }\end{array}$ & hojas & Anopheles gambiae s.l. Giles & Diallo et al. (2001) \\
\hline Aizoaceae & $\begin{array}{l}\text { D.C. } \\
\text { Glinus oppositifolius (L.) }\end{array}$ & hojas & Culex quinquefasciatus Say & Diallo et al. (2001) \\
\hline Aizoaceae & D.C. & hojas & Aedes aegypti (L.) & Diallo et al. (2001) \\
\hline Anacardiaceae & Lannea velutina A. Rich & hojas & Anopheles gambiae s.1. Giles & Diallo et al. (2001) \\
\hline Anacardiaceae & Lannea velutina A. Rich & hojas & Culex quinquefasciatus Say & Diallo et al. (2001) \\
\hline Anacardiaceae & Lannea velutina A. Rich & hojas & Aedes aegypti (L.) & Diallo et al. (2001) \\
\hline Anacardiaceae & Pistacia lentiscus L. & hojas & Culex pipiens molestus Forskal & Traboulsi et al. (2002) \\
\hline Anacardiaceae & Schinus molle L. & hojas & Culex quinquefasciatus Say & Iannacone et al. (2002) \\
\hline Annonaceae & Annona cherimolia Miller & semillas & Anopheles sp. & Bobadilla et al. (2002) \\
\hline Annonaceae & Annona muricata $\mathrm{L}$. & semillas & Anopheles sp. & Bobadilla et al. (2002) \\
\hline Annonaceae & Annona squamosa $\mathrm{L}$. & hojas & Culex quinquefasciatus Say & Jaswanth et al. (2003) \\
\hline Apiaceae & Apium graveolens $\mathrm{L}$. & semillas & Aedes aegypti (L.) & Nomin \& Nair (2001) \\
\hline Apiaceae & Daucus carota L. & semillas & Aedes aegypti (L.) & Momin et al. (2002) \\
\hline Apocynaceae & Rhazya stricta Decne & hojas & Culex pipiens $\mathrm{L}$. & El Hag et al. (1999) \\
\hline Araliaceae & Cussonia barteri Seem & hojas & Anopheles gambiae s.1. Giles & Diallo et al. (2001) \\
\hline
\end{tabular}


Ecol. apl. Vol. $3 \mathrm{~N}^{\mathrm{o}} 1$ y 2, pp. 64-72

\begin{tabular}{|c|c|c|c|c|}
\hline Araliaceae & Cussonia barteri Seem & hojas & Culex quinquefasciatus Say & Diallo et al. (2001) \\
\hline Araliaceae & Cussonia barteri Seem & hojas & Aedes aegypti (L.) & Diallo et al. (2001) \\
\hline Asteraceae & $\begin{array}{l}\text { Spilanthes acmella Murr. } \\
\text { Alnus glutinosa (L.) }\end{array}$ & hojas & Culex quinquefasciatus Say & Pitasawat et al. (1998) \\
\hline Betulaceae & $\begin{array}{l}\text { Gaertn } \\
\text { Alnus glutinosa (L.) }\end{array}$ & hojas & Aedes albopictus (Skuse) & David et al. (2000) \\
\hline Betulaceae & $\begin{array}{l}\text { Gaertn } \\
\text { Cordia curassavica (Jacq.) }\end{array}$ & hojas & Culex pipiens $\mathrm{L}$. & David et al. (2000) \\
\hline Boraginaceae & Roem \& Schutt & raíces & Aedes aegypti (L.) & Ioset et al. (2000) \\
\hline Euphorbiaceae & Manihot utilissima Pohl. & hojas & Aedes aegypti (L.) & Chariandy et al. (1999) \\
\hline Fabaceae & Cassia obtusifolia $\mathrm{L}$. & semillas & $\begin{array}{l}\text { Aedes aegypti }(\mathrm{L} .) \\
\text { Culex pipiens pallens }\end{array}$ & Jang et al. (2002) \\
\hline Fabaceae & Cassia obtusifolia L. & semillas & Coquillett & Jang et al. (2002) \\
\hline Fabaceae & Cassia tora $\mathrm{L}$. & semillas & $\begin{array}{l}\text { Aedes aegypti }(\mathrm{L} .) \\
\text { Culex pipiens pallens }\end{array}$ & Jang et al. (2002) \\
\hline Fabaceae & $\begin{array}{l}\text { Cassia tora } \mathrm{L} . \\
\text { Lonchocarpus chiricanus }\end{array}$ & semillas & Coquillett & Jang et al. (2002) \\
\hline Fabaceae & $\begin{array}{l}\text { Pittier } \\
\text { Vicia tetrasperma }(\mathrm{L} .)\end{array}$ & raíces & Aedes aegypti (L.) & Ioset et al. (2001) \\
\hline Fabaceae & $\begin{array}{l}\text { Schreber } \\
\text { Vicia tetrasperma }(\mathrm{L} .)\end{array}$ & semillas & $\begin{array}{l}\text { Aedes aegypti }(\mathrm{L} .) \\
\text { Culex pipiens pallens }\end{array}$ & Jang et al. (2002) \\
\hline Fabaceae & $\begin{array}{l}\text { Schreber } \\
\text { Quercus lusitanica var. }\end{array}$ & semillas & Coquillett & Jang et al. (2002) \\
\hline Fagaceae & $\begin{array}{l}\text { infectoria (G. Olivier) A. } \\
\text { DC }\end{array}$ & $\begin{array}{l}\text { corteza- } \\
\text { tronco }\end{array}$ & Culex pipiens $\mathrm{L}$. & Redwane et al. (2002) \\
\hline Fagaceae & Quercus robur L. & hojas & Aedes albopictus (Skuse) & David et al. (2000) \\
\hline Fagaceae & $\begin{array}{l}\text { Quercus robur } \mathrm{L} . \\
\text { Pelargonium citrosa Van }\end{array}$ & hojas & Culex pipiens $\mathrm{L}$. & David et al. (2000) \\
\hline Geraniaceae & $\begin{array}{l}\text { Leenii } \\
\text { Delphinium } x \text { cultorum }\end{array}$ & hojas & Anopheles stephensi Liston & Jeyabalan et al. (2003) \\
\hline Labiaceae & $\begin{array}{l}\text { Voss } \\
\text { Hyptus martiusii Mart ex }\end{array}$ & flores & Aedes aegypti (L.) & Miles et al. (2000) \\
\hline Labiaceae & Benth & hojas & Aedes aegypti (L.) & Araujo et al. (2003) \\
\hline Labiaceae & Lavandula stoechas $\mathrm{L}$. & hojas & Culex pipiens molestus Forskal & Traboulsi et al. (2002) \\
\hline Labiaceae & $\begin{array}{l}\text { Mentha microphylla Koch } \\
\text { Minthostachys setosa }\end{array}$ & hojas & Culex pipiens molestus Forskal & Traboulsi et al. (2002) \\
\hline Labiaceae & (Briq.) Epling & hojas & Aedes aegypti (L.) & Ciccia et al. (2000) \\
\hline Labiaceae & $\begin{array}{l}\text { Origanum syriacum } \mathrm{L} . \\
\text { Thymus capitatus }(\mathrm{L} .)\end{array}$ & hojas & Culex pipiens molestus Forskal & Traboulsi et al. (2002) \\
\hline Labiaceae & Hoffm. \& Link & hojas & Culex pipiens $\mathrm{L}$. & Mansour et al. (2000) \\
\hline Lauraceae & Persea americana Mill. & fruto & Aedes aegypti (L.) & Oberlies et al. (1998) \\
\hline Magnoliaceae & Illicium verum Hook & hojas & Culex quinquefasciatus Say & Pitasawat et al. (1998) \\
\hline Malvaceae & $\begin{array}{l}\text { Pavonia zeylanica L. } \\
\text { Azadirachta indica Adr. }\end{array}$ & hojas & Culex quinquefasciatus Say & Vahitha et al. (2002) \\
\hline Meliaceae & $\begin{array}{l}\text { Juss } \\
\text { Azadirachta indica Adr. }\end{array}$ & hojas & Anopheles stephensi Liston & Siddiqui et al. (2003) \\
\hline Meliaceae & $\begin{array}{l}\text { Juss } \\
\text { Azadirachta indica Adr. }\end{array}$ & hojas & Culex pipiens $\mathrm{L}$. & El Hag et al. (1999) \\
\hline Meliaceae & Juss & hojas & Culex quinquefasciatus Say & Iannacone et al. (2002) \\
\hline $\begin{array}{l}\text { Meliaceae } \\
\text { Menispermacea }\end{array}$ & $\begin{array}{l}\text { Turraea wakefieldii } \\
\text { Abuta grandifolia (Mart.) }\end{array}$ & raíces & Anopheles gambiae s.1. Giles & Ndung'uM et al. (2003) \\
\hline $\mathrm{e}$ & Sandwith & hojas & Aedes aegypti (L.) & Ciccia et al. (2000) \\
\hline Mimosaceae & Acacia ferruginea D.C. & hojas & Culex quinquefasciatus Say & Vahitha et al. (2002) \\
\hline Myrtaceae & $\begin{array}{l}\text { Myrtus communis } \mathrm{L} . \\
\text { Syzygium aromaticum }\end{array}$ & hojas & Culex pipiens molestus Forskal & Traboulsi et al. (2002) \\
\hline Myrtaceae & (Gaertn) Linn. & $\begin{array}{l}\text { hojas } \\
\text { hojas y }\end{array}$ & Culex pipiens $\mathrm{L}$. & El Hag et al. (1999) \\
\hline Orchidaceae & Vanilla fragans Ames & tronco & $\begin{array}{l}\text { Aedes aegypti }(\mathrm{L} .) \\
\text { Culex pipiens pallens }\end{array}$ & Sun et al. (2001) \\
\hline Piperaceae & Piper longum L. & fruto & Coquillett & Lee $(2000)$ \\
\hline Piperaceae & Piper nigrum L. & fruto & Aedes aegypti (L.) & Yang et al. (2002) \\
\hline Piperaceae & Piper nigrum L. & fruto & $\begin{array}{l}\text { Aedes togoi Theobald } \\
\text { Culex pipiens pallens }\end{array}$ & Park et al. (2002) \\
\hline Piperaceae & Piper nigrum $\mathrm{L}$. & fruto & Coquillett & Park et al. (2002) \\
\hline Piperaceae & Pothomorphe peltata L. & hojas & Aedes aegypti (L.) & Mongelli et al. (2002) \\
\hline
\end{tabular}


EFECTO INSECTICIDA DE Paullinia clavigera var. bullata Y Tradescantia zebrina EN EL CONTROL DE Anopheles benarrochi, VECTOR DE MALARIA EN UCAYALI, PERÚ

Diciembre 2004

\begin{tabular}{|c|c|c|c|c|}
\hline Rosaceae & Quillaja saponaria Molina & $\begin{array}{l}\text { corteza- } \\
\text { tronco } \\
\text { corteza- }\end{array}$ & Aedes aegypti (L.) & Pelah et al. (2002) \\
\hline Rosaceae & $\begin{array}{l}\text { Quillaja saponaria Molina } \\
\text { Feronia limonia }(\text { L.) }\end{array}$ & tronco & Culex pipiens $\mathrm{L}$. & Pelah et al. (2002) \\
\hline Rutaceae & $\begin{array}{l}\text { Swingle } \\
\text { Feronia limonia (L.) }\end{array}$ & hojas & Culex quinquefasciatus Say & Rahuman et al. (2000) \\
\hline Rutaceae & $\begin{array}{l}\text { Swingle } \\
\text { Feronia limonia (L.) }\end{array}$ & hojas & Anopheles stephensi Liston & Rahuman et al. (2000) \\
\hline Rutaceae & Swingle & hojas & Aedes aegypti (L.) & Rahuman et al. (2000) \\
\hline Rutaceae & $\begin{array}{l}\text { Melicope subunifoliata } \\
\text { (Stapf) T.G. Hartley }\end{array}$ & $\begin{array}{l}\text { corteza- } \\
\text { tronco }\end{array}$ & Aedes aegypti (L.) & Hung et al. (2003) \\
\hline Salicaceae & Populus nigra L. & hojas & Aedes albopictus (Skuse) & David et al. (2000) \\
\hline Salicaceae & Populus nigra $\mathrm{L}$. & $\begin{array}{l}\text { hojas } \\
\text { corteza- }\end{array}$ & Culex pipiens $\mathrm{L}$. & David et al. (2000) \\
\hline Sterculiaceae & Mansonia gagei Drumm & tronco & Aedes aegypti (L.) & Tiew et al. (2003) \\
\hline Thymelaeaceae & Dirca palustris L. & semillas & Aedes aegypti (L.) & Ramsewark et al. (2001) \\
\hline Tiliaceae & Microcos paniculata $\mathrm{L}$. & hojas & Aedes aegypti (L.) & Bandara et al. (2000) \\
\hline Verbenaceae & $\begin{array}{l}\text { Lantana camara } \mathrm{L} \text {. } \\
\text { Stachytarpheta }\end{array}$ & hojas & Culex quinquefasciatus Say & Iannacone et al. (2002) \\
\hline Verbenaceae & $\begin{array}{l}\text { jamaicensis (L.) Vaht } \\
\text { Kaempferia galanga }\end{array}$ & hojas & Aedes aegypti (L.) & Chariandy et al. (1999) \\
\hline Zingiberaceae & Kencur & hojas & Culex quinquefasciatus Say & Pitasawat et al. (1998) \\
\hline
\end{tabular}

\footnotetext{
${ }^{1}$ Instituto de Investigaciones de la Amazonía Peruana - Ucayali. Jr. Progreso 102, Pucallpa, Ucayali, Perú. Email: jfalcon36@hotmail.com / dperez@iiap.org.pe

${ }^{2}$ Laboratorio de Ecofisiología Animal. Facultad de Ciencias Naturales y Matemáticas. Universidad Nacional Federico Villarreal. Calle San Marcos 383, Pueblo Libre, Lima, Perú. E-mail: joseiannacone@hotmail.com
} 